\title{
Experimental Study on a Retrodirective System Utilizing Harmonic Reradiation from Rectenna
}

\author{
Tomohiko MITANI $^{\dagger \mathrm{a})}$, Member , Shogo KAWASHIMA ${ }^{\dagger *}$, Nonmember, and Naoki SHINOHARA ${ }^{\dagger b)}$, Member $^{\circ}$
}

\begin{abstract}
SUMMARY A retrodirective system utilizing harmonic reradiation from a rectenna is developed and verified for long-range wireless power transfer applications, such as low-power or battery-less devices and lightweight aerial vehicles. The second harmonic generated by the rectifying circuit is used instead of a pilot signal, and thus an oscillator for creating the pilot signal is not required. The proposed retrodirective system consists of a $2.45 \mathrm{GHz}$ transmitter with a two-element phased array antenna, a $4.9 \mathrm{GHz}$ direction-of-arrival (DoA) estimation system, a phase control system, and a rectenna. The rectenna, consisting of a half-wave dipole antenna, receives microwave power from the $2.45 \mathrm{GHz}$ transmitter and reradiates the harmonic toward the $4.9 \mathrm{GHz}$ DoA estimation system. The rectenna characteristics and experimental demonstrations of the proposed retrodirective system are described. From measurement results, the dc output power pattern for the developed retrodirective system is in good agreement with that obtained using manual beam steering. The measured DoA estimation errors are within the range of $-2.4^{\circ}$ to $4.8^{\circ}$.

key words: wireless power transfer, rectenna, harmonics reradiation, retrodirective system, direction-of-arrival estimation
\end{abstract}

\section{Introduction}

Long-range wireless power transfer (WPT) via radio waves, especially microwaves, has been studied and developed since the late 20th century [1], [2] for applications such as solar power satellites [3]-[5] and terrestrial WPT systems [2], [6]. Recently, WPT and radio-frequency (rf) energy harvesting have attracted a lot of attention as wireless power sources for Internet of Things devices and low-power sensors in wireless networks [6]-[8].

Retrodirective beam control is an essential technology for accurate, efficient, and stable long-range WPT to target devices [9], [10]. In most recent long-range WPT systems, the phased array technique is adopted for wireless power beaming to the receiving system. To determine the direction of the receiving site, a pilot signal is usually transmitted from the WPT receiving (Rx) system to the WPT transmitting (Tx) system. Then, the direction of arrival (DoA) of the pilot signal is estimated, and wireless power is beamed to the WPT receiving system from the WPT transmitting phased array system. Deterioration of retrodirectivity, which is mainly caused by the DoA estimation error and the beam direction error of the phased array, leads to degradation of

\footnotetext{
Manuscript received January 30, 2019.

Manuscript revised May 18, 2019.

${ }^{\dagger}$ The authors are with Research Institute for Sustainable Humanosphere, Kyoto University, Uji-shi, 611-0011 Japan.

*Presently, with NHK (Japan Broadcasting Corporation).

a)E-mail: mitani@rish.kyoto-u.ac.jp

b)E-mail: shino@rish.kyoto-u.ac.jp

DOI: 10.1587/transele.2019MMP0004
}

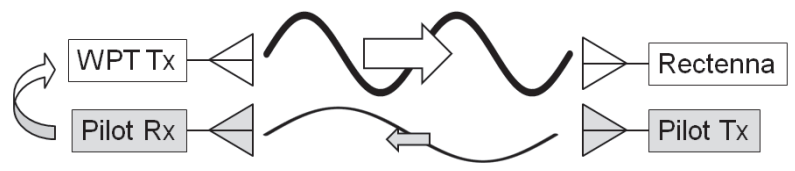

(a) Conventional retrodirective system

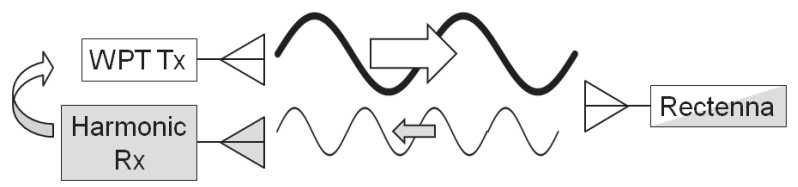

(b) Proposed retrodirective system

Fig. 1 Schematic diagrams of retrodirective systems for long-range WPT.

the transfer efficiency and interference with other communication systems.

The present study proposes a retrodirective system utilizing harmonic reradiation from a rectenna. In some WPT applications, such as low-power or battery-less devices and lightweight aerial vehicles, there is a strong possibility that the components required for creating a pilot signal cannot be installed in the WPT receiving system due to power and weight considerations. Figure 1 shows schematic diagrams of a conventional retrodirective system and the proposed system. The conventional system requires a pilot signal at the receiving site to determine the direction of the rectenna. In the proposed system, harmonics generated from the rectifier are used instead of the pilot signal. The direction of the rectenna can be estimated from the harmonics during WPT.

The novelty of the proposed system is that the nonlinearity of the rectifying operation is proactively utilized without any additional circuits or components. A similar concept on DOA estimation techniques has been studied for detecting RFID tags [11]. A DoA estimation technique utilizing back-scattering from RFID was studied [12], and a batteryless trackable device that used a ring oscillator has been also developed [13]. Compared with these previous studies, the rectenna itself provides the pilot signal directly by utilizing the reradiation of harmonics in the proposed system.

In the present paper, we verifies the proposed retrodirective system utilizing harmonic reradiation from a rectenna by experimental demonstrations. The rectenna characteristics used in the experiments are also described as a function of received microwave power density. 


\section{Rectenna Characteristics as a Function of Received Microwave Power Density}

\subsection{Rectenna Configuration}

We used a $2.45 \mathrm{GHz}$ rectenna consisting of a half-wave dipole antenna and a Schottky barrier diode package, Avago HSMS-286C, which behaves as a voltage doubler. A rectenna photograph is shown in Fig. 2. The rectenna was fabricated on a liquid crystal substrate Panasonic R-F705T, with the substrate thickness of $50 \mu \mathrm{m}$ and the copper thickness of $12 \mu \mathrm{m}$. The relative permittivity and loss tangent of the substrate are 3 and 0.0008 , respectively, at $2 \mathrm{GHz}$. The branch of the half-wave dipole antenna was $29 \mathrm{~mm}$ in length and $2 \mathrm{~mm}$ in width. A $1 \mathrm{nF}$ capacitor Murata GRM188B11H102KA01D was used as the smoothing capacitor. The other layout parameters can be found in [14].

In the previous study on the antenna patterns of harmonic reradiation from the rectenna shown in Fig. 2, the second harmonic reradiation was more effective to the broadside direction than the third harmonic [15]. The second harmonic was therefore used as the alternative pilot signal for the proposed retrodirective system. Basic rectenna characteristics, the rf-dc conversion efficiency and the second harmonic reradiated power, were measured at a fixed power density of $0 \mathrm{dBm} / \mathrm{cm}^{2}$ in the receiving area [14]. Although it was not designed for enhancing the second harmonic reradiation, the rectenna can be used to prove the validity of a retrodirective system utilizing harmonic reradiation. In the present paper, the rectenna characteristis are investigated as a function of received microwave power density for the purpose of designing the proposed retrodirective system.

\subsection{Measurement Configurations}

Figure 3 shows schematic diagrams of rectenna measurements. First, the received microwave power density $W_{\text {rf }}$ in the receiving area was measured as shown in Fig. 3(a). Then, the rectenna characteristics including the second harmonic reradiated power $P_{2 \mathrm{r}}$ in the transmitting area were measured as a function of $W_{\mathrm{rf}}$, as shown in Fig. 3 (b).

The WPT transmitter consisted of a signal generator (Agilent N5181A), a $100 \mathrm{~W}$ power amplifier (R\&K 24255050R), and a standard horn antenna (Scientific Atlanta 12-

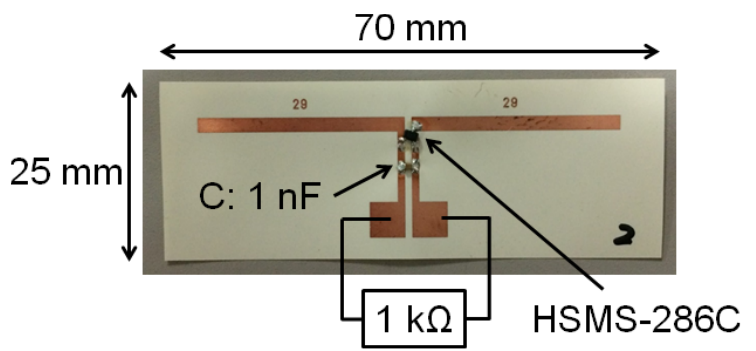

Fig. 2 Photograph of the rectenna consisting of a half-wave dipole antenna, a Schottky barrier diode, and a smoothing capacitor.
1.7). The transmitting frequency was $2.45 \mathrm{GHz}$. The transmitting antenna gain $G_{\mathrm{t}}$ was $16.9 \mathrm{dBi}$ at $2.45 \mathrm{GHz}$, and the transmitting distance $D$ was fixed at $4.6 \mathrm{~m}$. Then $W_{\text {rf }}$ can be estimated from the following equation:

$$
W_{\mathrm{rf}}=\frac{1}{4 \pi D^{2}} G_{\mathrm{t}} P_{\mathrm{t}},
$$

where $P_{\mathrm{t}}$ is the transmitting power. The microwaves were received by a standard dipole antenna (Antenna Giken ASD2425B) in the receiving area, and measured by a spectrum analyzer (Agilent E4440A). Then, $W_{\text {rf }}$ can be also obtained from the following equation:

$$
W_{\mathrm{rf}}=\frac{4 \pi}{\lambda_{1}^{2} G_{\mathrm{r}}} P_{\mathrm{r}},
$$

where $\lambda_{1}, G_{\mathrm{r}}$ and $P_{\mathrm{r}}$ are the wavelength of free space at $2.45 \mathrm{GHz}\left(\lambda_{1} \approx 12.24 \mathrm{~cm}\right)$, the receiving antenna gain and the received power, recpectively. In the measurements, we obtained $W_{\mathrm{rf}}$ from Eq. (2) by using the measured $P_{\mathrm{r}}$, and confirmed the values of $W_{\text {rf }}$ from Eq. (1).

After the power density measurements, the standard dipole antenna was replaced with the rectenna for measuring the rectenna characteristics, as shown in Fig. 3(b). The rf-dc conversion efficiency $\eta_{1}$ was obtained as follows:

$$
\eta_{1}=\frac{P_{\mathrm{dc}}}{P_{\mathrm{rf}}}
$$

where $P_{\mathrm{dc}}$, and $P_{\mathrm{rf}}$ are the dc output power and the microwave input power to the rectenna, respectively. $P_{\mathrm{dc}}$ was obtained from the measured dc voltage $V_{\mathrm{dc}}$ by a multimeter (Agilent 34401A). The output load $R_{\mathrm{L}}$ of the rectenna was fixed at $1 \mathrm{k} \Omega$ in the present study. Then $P_{\mathrm{dc}}$ is expressed as

$$
P_{\mathrm{dc}}=\frac{V_{\mathrm{dc}}^{2}}{R_{\mathrm{L}}}
$$

$P_{\mathrm{rf}}$ was obtained by replacing the variables $G_{\mathrm{r}}$ and $P_{\mathrm{r}}$ in Eq. (2) with the antenna gain of the rectenna $G_{1 \mathrm{r}}$ for the fundamental and $P_{\mathrm{rf}}$, as follows:

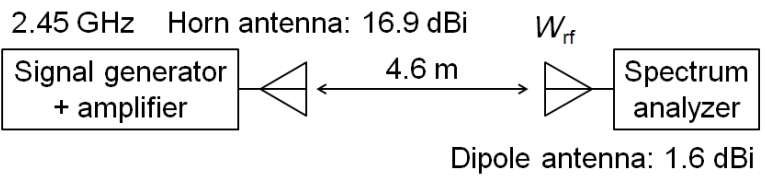

(a) Measurements of the received microwave power density $W_{\mathrm{rf}}$ in the receiving area

$2.45 \mathrm{GHz}$ Horn antenna: $16.9 \mathrm{dBi}$

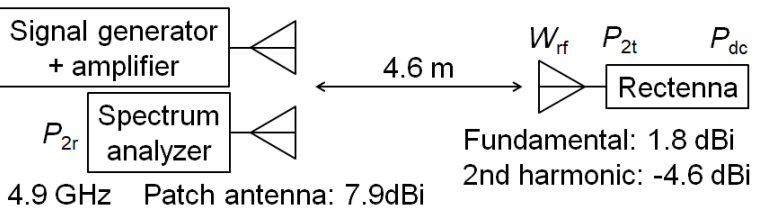

(b) Measurements of the rectenna characteristics

Fig. 3 Measurement configurations of the rectenna characteristics. 


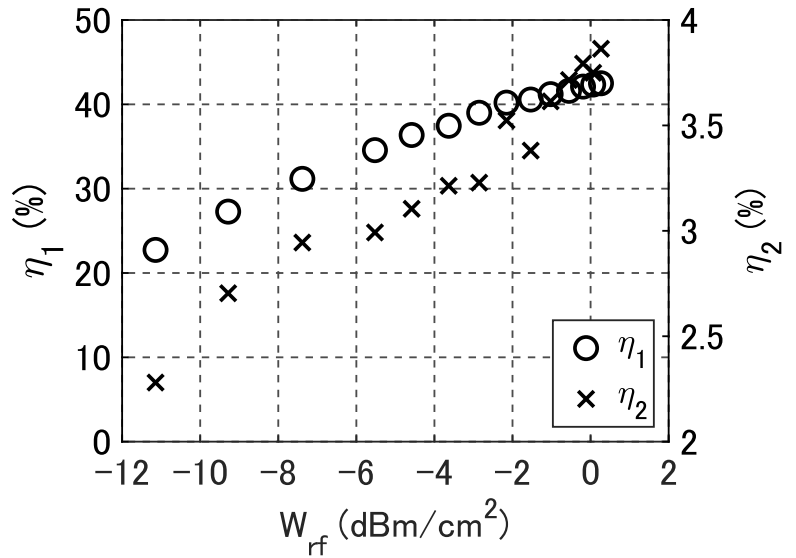

Fig. 4 Measurement results of the rf-dc conversion efficiency $\eta_{1}$ and the second harmonic reradiation efficiency $\eta_{2}$ of the rectenna to the microwave power density $W_{\mathrm{rf}}$ in the receiving area.

$$
P_{\mathrm{rf}}=\frac{\lambda_{1}^{2}}{4 \pi} G_{1 \mathrm{r}} W_{\mathrm{rf}} .
$$

We assumed $G_{1 \mathrm{r}}$ was $1.8 \mathrm{dBi}$, which was calculated by the electromagnetic simulation software CST STUDIO SUITE, as it couldn't be measured directly.

The received second harmonic reradiated power $P_{2 \mathrm{r}}$ in the transmitting area was measured simultaneously during the measurements of rectenna characteristics as shown in Fig. 3 (b). The second harmonic measurement system consisted of a fabricated rectangular patch antenna at $4.9 \mathrm{GHz}$ and a spectrum analyzer. The patch antenna gain $G_{2 \mathrm{r}}$ was $7.9 \mathrm{dBi}$. Then we can estimate the second harmonic reradiated power $P_{2 \mathrm{t}}$ from the rectenna by the following Friis equation:

$$
P_{2 \mathrm{t}}=\left(\frac{4 \pi D}{\lambda_{2}}\right)^{2} \frac{P_{2 \mathrm{r}}}{G_{2 \mathrm{t}} G_{2 \mathrm{r}}},
$$

where $\lambda_{2}$ and $G_{2 t}$ are the wavelength of free space at $4.9 \mathrm{GHz}\left(\lambda_{2} \approx 6.12 \mathrm{~cm}\right)$ and the antenna gain of the rectenna for the second harmonic, respectively. We assumed $G_{2 \mathrm{t}}$ was $-4.6 \mathrm{dBi}$, which was also obtained by the electromagnetic simulations. Finally, the second harmonic reradiation efficiency $\eta_{2}$ can be obtained as follows:

$$
\eta_{2}=\frac{P_{2 \mathrm{t}}}{P_{\mathrm{rf}}}
$$

\subsection{Results and Discussion}

Measurement results of the rf-dc conversion efficiency $\eta_{1}$ and the second harmonic reradiation efficiency $\eta_{2}$ to the microwave power density $W_{\mathrm{rf}}$ in the receiving area are shown in Fig. 4. The maximum $\eta_{1}$ was $42.5 \%$ at $W_{\mathrm{rf}}=$ $0.3 \mathrm{dBm} / \mathrm{cm}^{2}$, which is comparable with the measurement results in the previous study [14], the maximum $\eta_{1}=47.4 \%$ at $W_{\text {rf }}=0 \mathrm{dBm} / \mathrm{cm}^{2}$ and $R_{\mathrm{L}}=1.5 \mathrm{k} \Omega$. The slight degradation of $\eta_{1}$ was attributed to the change of $R_{\mathrm{L}} . \eta_{2}$ was estimated from $2.3 \%(-16.4 \mathrm{~dB})$ to $3.9 \%(-14.1 \mathrm{~dB})$, which is

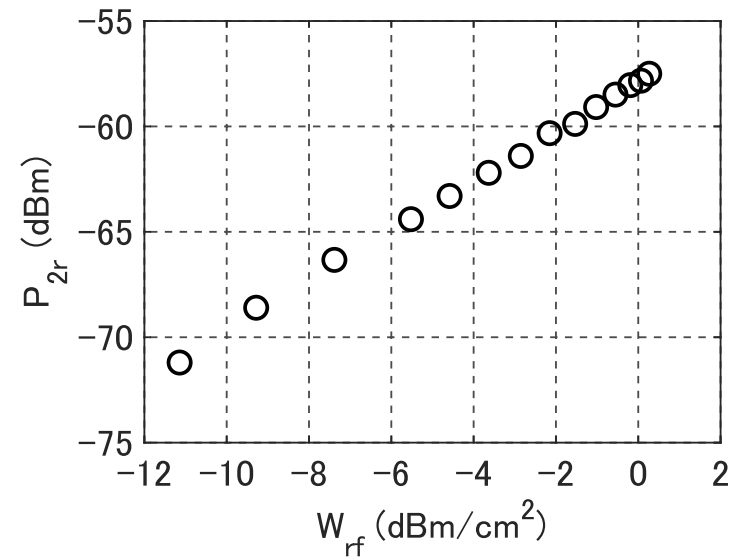

Fig. 5 Measurement results of the received second harmonic reradiated power $P_{2 \mathrm{r}}$ at the transmitting position to the microwave power density $W_{\mathrm{rf}}$ in the receiving area.

comparable or a little worse than the conversion loss of commercially available frequency doubler in microwave bands (e.g. [16]).

Measurement results of the received second harmonic reradiated power $P_{2 \mathrm{r}}$ in the transmitting area to the microwave power density $W_{\mathrm{rf}}$ at the receiving position are shown in Fig. 5. It was found that $P_{2 \mathrm{r}}$ was almost a linear function of $W_{\mathrm{rf}}$. The applicable power level for the DoA estimation system, which is described in Sect. 3, was $-69 \mathrm{dBm}$, measured in the previous study [17]. When $P_{2 \mathrm{r}}$ is lower than $-69 \mathrm{dBm}$, the phase detector in the DoA estimation system becomes disabled and it outputs meaningless values. Therefore the condition $P_{2 \mathrm{r}}>-69 \mathrm{dBm}$, i.e. $W_{\text {rf }}>-9.7 \mathrm{dBm} / \mathrm{cm}^{2}$ and $P_{\text {rf }}>2.9 \mathrm{dBm}$, is mandatory for the stable operation of the proposed retrodirective system in the present study.

\section{Configurations of the Proposed Retrodirective Sys- tem}

Figure 6 shows the experimental configuration of the retrodirective system. The $2.45 \mathrm{GHz}$ WPT transmitter was set on a turntable. The midpoint between the two transmitting H-plane horn antennas was located on the rotation axis of the turntable. The rotation angle $\theta$ of the turntable corresponded to the direction of the transmitting antennas. The 4.9 GHz DoA estimation system was mounted above the $2.45 \mathrm{GHz}$ WPT transmitter for receiving the second harmonic radiated from a rectenna. The midpoint between the two receiving rectangular patch antennas was located on the rotation axis of the turntable. The direction of the receiving antennas was brought in line with that of the transmitting antennas. The phase difference between the two receiving antennas was sent to the phase control system, which converted the phase difference data to phase shift data for the two transmitting antennas. The rectenna was placed at a distance of $4.6 \mathrm{~m}$ from the WPT transmitter. The output load of the rectenna was $1 \mathrm{k} \Omega$. A photograph of the overall configuration is shown in Fig. 7. The experiments were conducted 


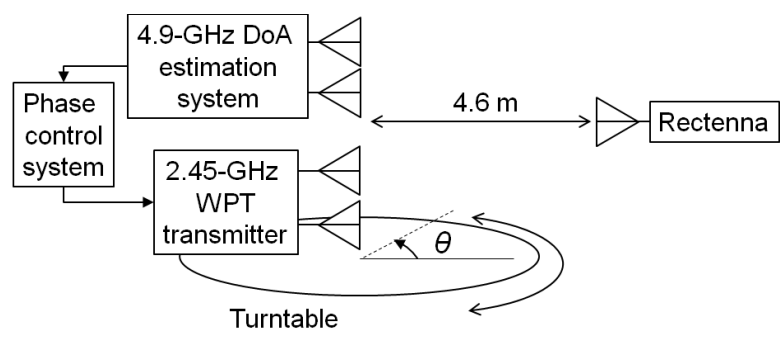

Fig. 6 Schematic diagram of the experimental configuration of the retrodirective system. $\theta$ is the rotation angle of the turntable.

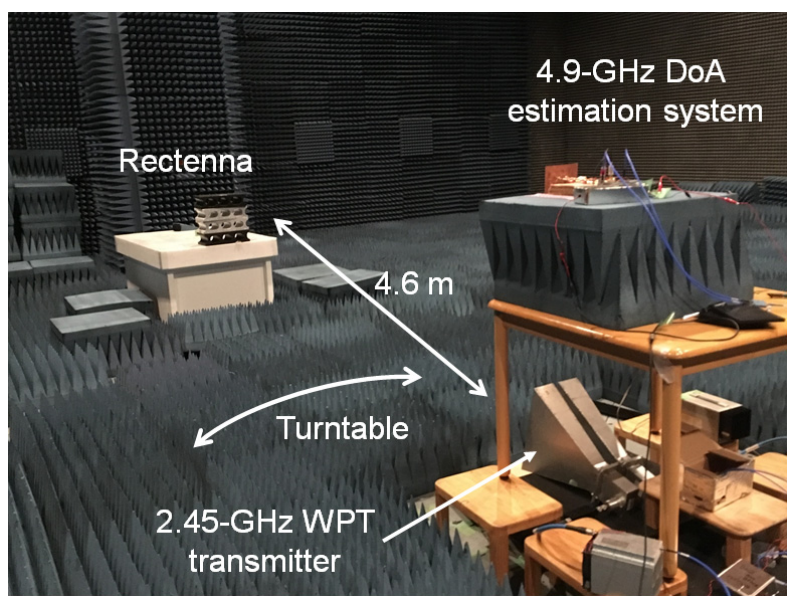

Fig. 7 Photograph of the experimental configuration of the retrodirective system.

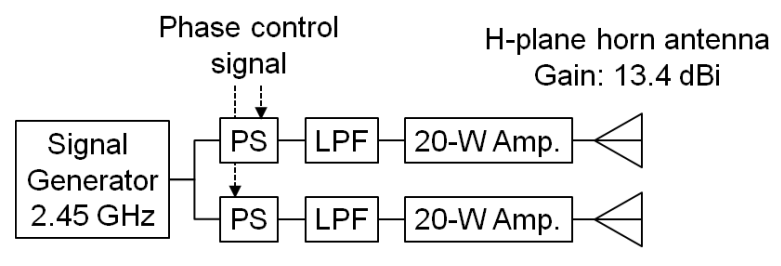

Fig. 8 Schematic of the $2.45 \mathrm{GHz}$ WPT transmitter.

in an anechoic chamber.

Figure 8 shows a schematic of the $2.45 \mathrm{GHz}$ WPT transmitter. The $2.45 \mathrm{GHz}$ microwave was generated by the signal generator and divided into two ways. Each microwave went through the analog phase shifter (R\&K PS 2450) for beam steering, and the low pass filter (MiniCircuits SLP-2950+) for rejecting harmonics. Then it was amplified to $20 \mathrm{~W}$ by the power amplifier (R\&K CA2450BW100) and radiated through the H-plane horn antenna whose physical aperture was $9.9 \mathrm{~cm} \times 41 \mathrm{~cm}$ and whose antenna gain was $13.4 \mathrm{dBi}$. The antenna spacing was $10.4 \mathrm{~cm} \approx 0.85 \lambda_{1}$. The phase of the analog phase shifter was shifted by controlled voltage from 0 to $12 \mathrm{~V}$. Due to two phased array configuration, the phase of one transmitter was fixed and the phase of another transmitter was controlled by the phase control signal from the phase control system.

Figure 9 shows a schematic of the $4.9 \mathrm{GHz}$ DoA estimation system. The second harmonic from the rectenna

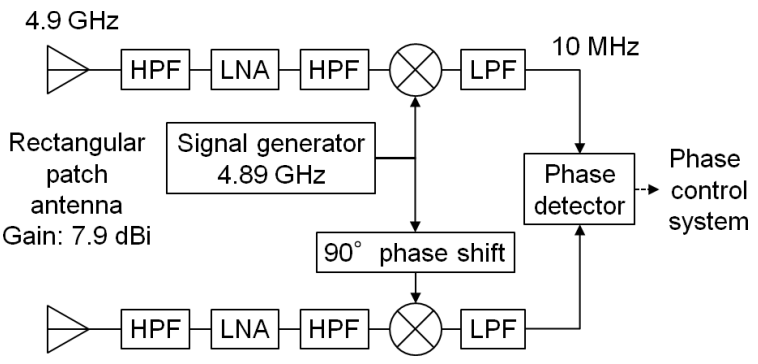

Fig. 9 Schematic of the $4.9 \mathrm{GHz}$ DoA estimation system.

was received by two rectangular patch antennas, and the phase difference was obtained by a simple interferometry method. The physical aperture of each patch antenna was $1.95 \mathrm{~cm} \times 1.77 \mathrm{~cm}$, and its antenna gain was $7.9 \mathrm{dBi}$, which is the same as the receiving antenna described in Sect. 2 . The antenna spacing was $6.0 \mathrm{~cm} \approx 0.49 \lambda_{2}$. The received harmonic was amplified by the low noise amplifier (MiniCircuits ZX-60-542LN+), before and after which the high pass filters (Mini-Circuits VHF-3800+) were inserted. Then the received harmonic was down-converted to $10 \mathrm{MHz}$ via the mixer (Mini-Circuits ZMX-7GR) and the low pass filter (Mini-Circuits SLP-250+). Finally, the phase detector (Analog Devices AD8302) output the voltage corresponding to the phase difference of two $10 \mathrm{MHz}$ signals, to the phase control system.

The phase control system consisted of a digital multimeter (TEXIO DL-2060), PC and data acquisition unit with analog output module (National Instruments NI cDAQ9172, NI-9263). The output voltage from the phase detector in the $4.9 \mathrm{GHz}$ DoA estimation system was measured by the multimeter and the PC converted the collected voltage data into the phase control signal by the software NI LabVIEW. In the process of data conversion, the calibration function of phase difference between the receiving antennas including mutual coupling effects was introduced. It was experimentally derived from the phase difference data of the conventional DoA estimation measurements. The derived calibration function $h(\delta)$ is expressed as the following fifth degree polynomial:

$$
\begin{aligned}
h(\delta)= & 4.50 \times 10^{-9} \delta^{5}+1.06 \times 10^{-7} \delta^{4} \\
& -7.27 \times 10^{-5} \delta^{3}-1.07 \times 10^{-3} \delta^{2} \\
& +0.273 \delta+1.39,
\end{aligned}
$$

where $\delta$ is the measured phase difference between the receiving antenna. The calibration function $h(\delta)$ was added to the measured phase difference data in the software NI LabVIEW. Finally, the data acquisition unit output the phase control signals to the phase shifters in the $2.45 \mathrm{GHz}$ WPT transmitter.

\section{Measurement Results of the Retrodirective System}

\subsection{Beam Pattern of the WPT Transmitting Antenna}

Before experimenting the retrodirective system, the beam 


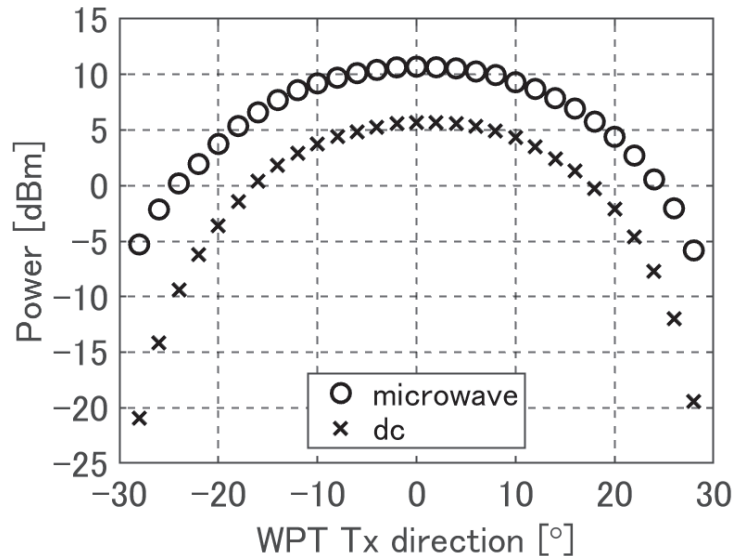

Fig. 10 Measured beam patterns of the WPT transmitting antenna (circle: beam pattern of the estimated microwave power $P_{\mathrm{rf}}$, cross: dc output power of rectenna $P_{\mathrm{dc}}$ ).

pattern of the $2.45 \mathrm{GHz}$ WPT transmitting antennas was measured by replacing the rectenna shown in Fig. 6 with a standard dipole antenna. Then the beam pattern of dc output power $P_{\mathrm{dc}}$ was also measured by monitoring the dc output voltage of rectenna. The phase difference between two transmitters was set to zero, and the antenna direction was changed from $-28^{\circ}$ to $28^{\circ}$ by the turntable.

Figure 10 shows measurement results of the beam patterns. The results of microwave beam pattern are indicated as estimated microwave power levels $P_{\mathrm{rf}}$ which the rectenna received. $P_{\mathrm{rf}}$ can be estimated from Eqs. (2) and (5), by using the measured received power $P_{\mathrm{r}}$. The estimated $P_{\mathrm{rf}}$ in the receiving area was $10.7 \mathrm{dBm}$ to the boresight direction when $\theta=0^{\circ}$, and it satisfied the operation condition $P_{\mathrm{rf}}>2.9 \mathrm{dBm}$, as described in Sect. 2. Hence, we confirmed that the transmitting power level was enough large to conduct experimental demonstrations of the proposed retrodirective system.

\subsection{Retrodirective Experiments}

Figure 11 shows measurement results of the retrodirective experiments when the transmitting antenna direction was changed from $-28^{\circ}$ to $28^{\circ}$ by the turntable. All the beam patterns were evaluated using the dc output power of the rectenna. "Retrodirective" indicates the results of overall retrodirective system utilizing the harmonic reradiation. The received harmonic reradiation power $P_{2 \mathrm{r}}$ in the transmitting area was $-60.3 \mathrm{dBm}$ when $\theta=0^{\circ}$. "Manual beam control" indicates the results of manual beam steering by the transmitting phased array to the rectenna direction. In this measurements, the DoA estimation data was not referred to determining the beam direction. "No beam control" indicates the fixed beam pattern of the transmitting antenna to the broadside direction, which is the same as the beam pattern of dc output power in Fig. 10.

Figure 12 shows the averaged DoA estimation errors simultaneously obtained during the retrodirective system measurements. The DoA estimation error measurements

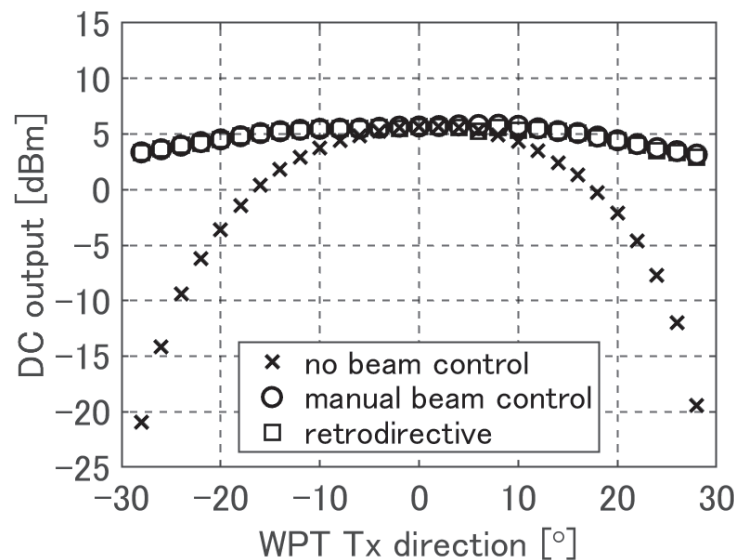

Fig. 11 Measured beam patterns of the retrodirective system (square), manual beam control (circle), and fixed beam pattern toward the broadside direction (cross).

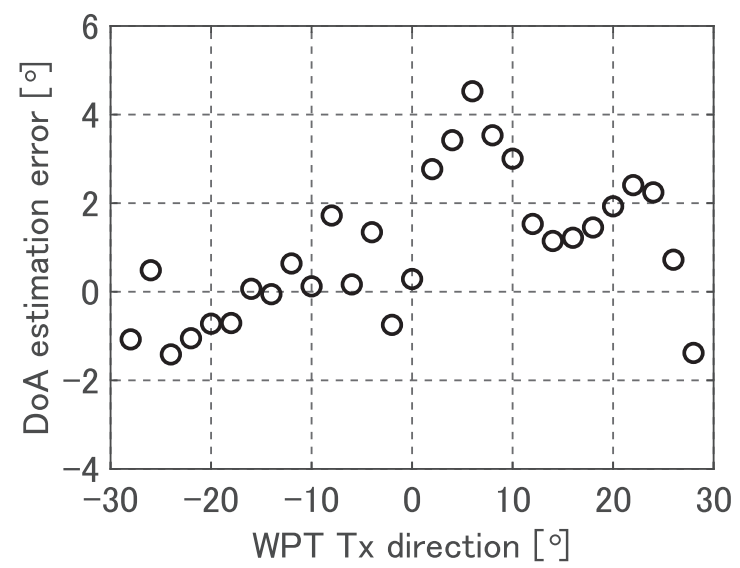

Fig. 12 Averaged DoA estimation errors (number of samples: 3 ) during the retrodirective system measurements.

were conducted three times. The true values of DoA coincided with the angles between the transmitting antenna direction and the boresight direction. Mutual coupling effects on the $4.9 \mathrm{GHz}$ receiving antennas, which were previously derived from the electromagnetic simulations [17], was subtracted in Fig. 12.

\section{Discussion}

The dc output power pattern of the retrodirective system was almost in agreement with that of manual beam steering, as shown in Fig. 11. This means the WPT transmitter in the retrodirective system automatically transmitted the microwave power to the direction of rectenna as well as the manual beam control.

The DoA estimation errors were within the range from $-2.4^{\circ}$ to $4.8^{\circ}$ from Fig. 12. The main cause of the DoA estimation errors was misalignment of the overall retrodirective system, since there was reproductivity in the measurements. In particular, the errors became lager when the transmitting antenna direction $\theta$ was small. The path length difference 
Table 1 Link budget of the developed retrodirective system utilizing harmonic reradiation in the boresight direction when $\theta=0^{\circ}$. * indicates that the data were calculated by electromagnetic simulations.

\begin{tabular}{llr}
\hline 2.45 GHz WPT & & \\
\hline Transmitting microwave power & $40 \mathrm{~W}$ & $46.0 \mathrm{dBm}$ \\
Transmitting antenna gain* & 43.7 & $16.4 \mathrm{dBi}$ \\
Free space loss $(4.6 \mathrm{~m}, 2.45 \mathrm{GHz})$ & $4.48 \times 10^{-6}$ & $53.5 \mathrm{~dB}$ \\
Receiving antenna gain of the rectenna* & 1.51 & $1.8 \mathrm{dBi}$ \\
Received power by the rectenna & $11.7 \mathrm{~mW}$ & $10.7 \mathrm{dBm}$ \\
\hline 4.9 GHz harmonic reradiation & & \\
\hline Received harmonic power & $0.93 \mathrm{nW}$ & $-60.3 \mathrm{dBm}$ \\
Receiving antenna gain* & 6.17 & $7.9 \mathrm{dBi}$ \\
Free space loss $(4.6 \mathrm{~m}, 4.9 \mathrm{GHz})$ & $1.12 \times 10^{-6}$ & $59.5 \mathrm{~dB}$ \\
Transmitting antenna gain of the rectenna* & 0.347 & $-4.6 \mathrm{dBi}$ \\
Reradiated harmonic power from the rectenna & $0.39 \mathrm{~mW}$ & $-4.1 \mathrm{dBm}$ \\
\hline
\end{tabular}

between two receiving antennas of the DoA estimation system was approximated to $2 d \sin \theta$, where $d$ is the antenna spacing, assuming $d / D \ll 1$ and $\sin \theta \ll 1$. When $\theta= \pm 2^{\circ}$ the path length difference is calculated to $2.1 \mathrm{~mm}$, which is small enough to cause the DoA errors by misalignment. Asymmetry of the DoA estimation errors with respect to the transmitting antenna direction is mainly due to the asymmetric calibration function $h(\delta)$ in Eq. (8). As $h(\delta)$ is asymmetric, the DoA estimation errors became asymmetric.

In addition, it was confirmed from the measurements that the interference with the direct incoming waves from the WPT transmitter to the phase detector of the $4.9 \mathrm{GHz}$ DoA estimation system was below the noise floor level. Also the errors of the phase detector were negligibly small, compared with the effects of misalignment. The phase measurement accuracy of the detector is $10 \mathrm{mV} /{ }^{\circ}$; whereas the noise level of detector output voltage was less than $5 \mathrm{mV}$.

In spite of the DoA estimation errors, the dc output power of the retrodirective system was sufficiently comparable to the manual beam steering. This is attributed to the beam width of the WPT transmitting array antenna. The half-power width and $1 \mathrm{~dB}$ width of the transmitting antenna can be calculated to $28^{\circ}$ and $17^{\circ}$, respectively, from Fig. 10. As the beam width was wider than the DoA estimation errors, there was no significant difference of beam patterns between the retrodirective system and manual beam steering.

From the discussions above, the developed retrodirective system utilizing harmonic reradiation succeeded as a new WPT retrodirective method. The reproductive DoA estimation errors due to misalignment can be calibrated again, and the effects can be added to the calibration function $h(\delta)$. In order to investigate the developed retrodirective system with higher accuracy, the beam width of the transmitting antenna should be sharper using a phased array with numerous elements.

\section{Conclusion}

A retrodirective system that utilizes harmonic reradiation was developed and verified. The proposed retrodirective system can be applied for long-range WPT applications such as low-power or battery-less devices and lightweight aerial vehicles as it does not require a pilot signal. The DoA estimation errors caused by misalignment, which are compensated for by a wide transmitting antenna beam width, can be calibrated in the retrodirective system.

The link budget of the developed retrodirective system utilizing harmonic reradiation in the boresight direction when $\theta=0^{\circ}$ is summarized in Table 1 . The link budget is classified into $2.45 \mathrm{GHz}$ WPT and $4.9 \mathrm{GHz}$ harmonic reradiation. Expansion of the WPT distance is a future work for more realistic WPT applications.

\section{Acknowledgments}

This work was supported in part by JSPS Kakenhi Grant Number 15K18051. Experiments were conducted thanks to collaborative research program: Microwave Energy Transmission Laboratory (METLAB), Research Institute for Sustainable Humanosphere, Kyoto University.

\section{References}

[1] W.C. Brown, "The History of Power Transmission by Radio Waves," IEEE Trans. Microw. Theory Techn., vol.32, no.9, pp.1230-1242, Sept. 1984. DOI: 10.1109/TMTT.1984.1132833

[2] N. Shinohara, "Power Without Wires," IEEE Microw. Mag., vol.102, no.11, pp.S64-S73, Dec. 2011. DOI: 10.1109/ MMM.2011.942732

[3] P.E. Glaser, "Power from the Sun: Its Future," Science, vol.162, no.3856, pp.857-861, Nov. 1968. DOI: $10.1126 /$ science.162.3856.857

[4] P. Jaffe and J. McSpadden, "Energy Conversion and Transmission Modules for Space Solar Power," Proc. IEEE, vol.101, no.6, pp.1424-1437, June 2013. DOI: 10.1109/JPROC.2013.2252591

[5] S. Sasaki, K. Tanaka, and K. Maki, "Microwave Power Transmission Technologies for Solar Power Satellites," Proc. IEEE, vol.101, no.6, pp.1438-1447, June 2013. DOI: 10.1109/JPROC.2013.2246851

[6] N. Shinohara, ed., Recent Wireless Power Transfer Technologies via Radio Waves, River Publishers, Gistrup, 2018.

[7] Z. Popović, E.A. Falkenstein, D. Costinett, and R. Zane, "Low-Power Far-Field Wireless Powering for Wireless Sensors," Proc. IEEE, vol.101, no.6, pp.1397-1409, June 2013. DOI: 10.1109/ JPROC.2013.2244053

[8] H.J. Visser and R.J.M. Vullers, "RF Energy Harvesting and Transport for Wireless Sensor Network Applications: Principles and Requirements," Proc. IEEE, vol.101, no.6, pp.1410-1423, June 2013. DOI: 10.1109/JPROC.2013.2250891

[9] B. Strassner and K. Chang, "Microwave Power Transmission: Historical Milestones and System Components," Proc. IEEE, vol.101, no.6, pp.1379-1396, June 2013. DOI: 10.1109/ JPROC.2013.2246132

[10] A. Massa, G. Oliveri, F. Viani, and P. Rocca, "Array Designs for Long-Distance Wireless Power Transmission: State-of-the-Art and Innovative Solutions," Proc. IEEE, vol.101, no.6, pp.1464-1481, June 2013. DOI: 10.1109/JPROC.2013.2245491

[11] M. Bolić, D. Simplot-Ryl, and I. Stojmenović, "RFID systems: research trends and challenges," John Wiley \& Sons, Chichester, 2010.

[12] N. Homma, K. Toda, and Y. Tsunekawa, "DoA Estimation Technique of Back-Scattering Signal from RFID for Gesture Recognition," Proc. 2015 9th European Conference on Antennas and Propagation, Lisbon, Portugal, April 2015.

[13] D. Belo, D.C. Ribeiro, P. Pinho, and N.B. Carvalho, "A Low Complexity and Accurate Battery-Less Trackable Device," Proc. 
2018 IEEE MTT-S Int. Microwave Symp, Philadelphia, USA, pp.1269-1271, June 2018.

[14] T. Mitani, S. Kawashima, and T. Nishimura, "Analysis of Voltage Doubler Behavior of 2.45-GHz Voltage-Doubler-Type Rectenna," IEEE Trans. Microw. Theory Techn., vol.65, no.4, pp.1051-1057, April 2017. DOI: 10.1109/TMTT.2017.2668413

[15] S. Kawashima, N. Shinohara, and T. Mitani, "Study on Rectenna Harmonics Reradiation for Microwave Power Transfer with a Harmonics-Based Retrodirective System," Proc. Int. Symp. Antennas Propag., pp.524-525, Okinawa, Japan, Oct. 2016.

[16] Mini Circutis, Frequency Multiplier ZX90-2-36+ Datasheet [Online]. Available: https://www.minicircuits.com/pdfs/ ZX90-2-36+.pdf

[17] T. Mitani, S. Kawashima, and N. Shinohara, "Direction-of-Arrival Estimation by Utilizing Harmonic Reradiation from Rectenna," Proc. 2018 IEEE MTT-S Wireless Power Transfer Conference, Montreal, Canada, June 2018.

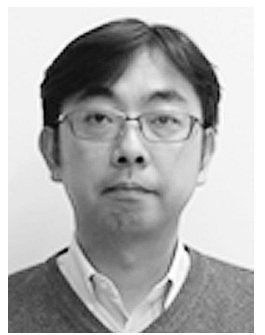

Tomohiko Mitani received the B.E. degree in electrical and electronic engineering, the M.E. degree in informatics, and the Ph.D. in electrical engineering from Kyoto University, Kyoto, Japan, in 1999, 2001, and 2006, respectively. He was an Assistant Professor with the Radio Science Center for Space and Atmosphere, Kyoto University, in 2003. He has been an Associate Professor with the Research Institute for Sustainable Humanosphere, Kyoto University, since 2012. His current research interests include microwave heating application and microwave power transfer. $\mathrm{He}$ is a member of the IEICE Technical Committee on Microwaves. He has been a board member of Japan Society of Electromagnetic Wave Energy Applications (JEMEA) since 2104. He was a treasurer of IEEE MTT-S Kansai Chapter from 2014 to 2017, and has been since 2019.

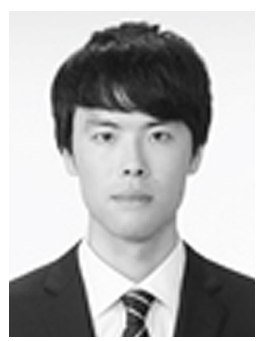

Shogo Kawashima received the B.E. degree in electrical and electronic engineering and the M.E. degree in electrical engineering from Kyoto University, Kyoto, Japan, in 2016 and 2018 , respectively. His research interests included microwave power transfer technology.

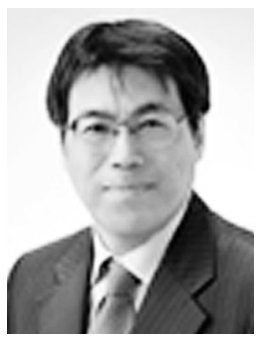

Naoki Shinohara received the B.E. degree in electronic engineering, the M.E. and Ph.D (Eng.) degrees in electrical engineering from Kyoto University, Japan, in 1991, 1993 and 1996, respectively. He was a research associate in the Radio Atmospheric Science Center, Kyoto University from 1996. He was a research associate of the Radio Science Center for Space and Atmosphere, Kyoto University by recognizing the Radio Atmospheric Science Center from 2000, and there he was an associate professor since 2001. He was an associate professor in Research Institute for Sustainable Humanosphere, Kyoto University by recognizing the Radio Science Center for Space and Atmosphere since 2004. From 2010, he has been a professor in Research Institute for Sustainable Humanosphere, Kyoto University. He has been engaged in research on Solar Power Station/Satellite and Microwave Power Transmission system. He is IEEE MTT-S Technical Committee 26 (Wireless Power Transfer and Conversion) chair, IEEE MTT-S Kansai Chapter TPC member, IEEE Wireless Power Transfer Conference advisory committee member, international journal of Wireless Power Transfer (Cambridge Press) executive editor, Radio Science for URSI Japanese committee $\mathrm{C}$ member, a memebr of the IEICE Technical Committee on Wireless Power Transfer, Wireless Power Transfer Consortium for Practical Applications (WiPoT) chair, and Wireless Power Management Consortium (WPMc) chair. 